\title{
Functional Properties of Synaptic Transmission in Primary Sense Organs
}

\author{
Joshua H. Singer, ${ }^{1}$ Elisabeth Glowatzki, ${ }^{2}$ Tobias Moser, ${ }^{3}$ Ben W. Strowbridge, ${ }^{4}$ Vikas Bhandawat, ${ }^{5}$ \\ and Alapakkam P. Sampath ${ }^{6}$ \\ ${ }^{1}$ Departments of Ophthalmology and Physiology, Northwestern University Feinberg School of Medicine, Chicago, Illinois 60611, ${ }^{2}$ Department of \\ Otolaryngology, The Johns Hopkins University School of Medicine, Baltimore, Maryland 21205, ${ }^{3}$ Department of Otolaryngology and Center for Molecular \\ Physiology of the Brain, University of Göttingen, 37073 Göttingen, Germany, ${ }^{4}$ Department of Neurosciences, Case Western Reserve University, Cleveland, \\ Ohio 44106, ${ }^{5}$ Department of Neurobiology, Harvard Medical School, Boston, Massachusetts 02115, and ${ }^{6}$ Zilkha Neurogenetic Institute, Department of \\ Physiology and Biophysics, University of Southern California, Los Angeles, California 90089
}

Sensory receptors transduce physical stimuli in the environment into neural signals that are interpreted by the brain. Although considerable attention has been given to how the sensitivity and dynamic range of sensory receptors is established, peripheral synaptic interactions improve the fidelity with which receptor output is transferred to the brain. For instance, synapses in the retina, cochlea, and primary olfactory system use mechanisms that fine-tune the responsiveness of postsynaptic neurons and the dynamics of exocytosis; these permit microcircuit interactions to encode efficiently the output of sensory receptors with the fidelity and dynamic range necessary to extract the salient features of the physical stimuli. The continuous matching of presynaptic and postsynaptic responsiveness highlight how the primary sensory organs have been optimized and can be modulated to resolve sparse sensory signals and to encode the entire range of receptor output.

\section{Introduction}

All sensory systems face a similar signal processing challenge: how to maximize sensitivity to detect sparse signals (e.g., a few odorant molecules or photons) while retaining the dynamic range sufficient to encode inputs that vary widely in intensity (e.g., the cochlea responds to sounds that vary in intensity $>100$ $\mathrm{dB}$ ). This challenge is overcome in part at the level of the sensory receptors themselves: receptors are sensitive (e.g., a single photon reliably changes the membrane potential of a rod photoreceptor) and numerous enough to capture stimuli arising in the physical world, and receptors possess intrinsic mechanisms to regulate their gain, or adapt, as stimulus intensities change.

Subcortical and subthalamic circuits process significantly sensory receptor output before it generates percepts. To ensure efficient signal coding, the cellular and network properties of these neural circuits must be well matched to the receptor output: i.e., sensory circuits must possess cellular processes that allow them to process a range of inputs of widely ranging strengths generated by varying numbers of receptors. Here, we highlight synaptic mechanisms exhibited by sensory receptors and their postsynaptic partners that allow primary sensory circuits to encode a broad

Received July 13, 2009; revised Aug. 11, 2009; accepted Aug. 13, 2009.

This work was supported by National Institutes of Health Grants EY17836 (J.H.S.), DC06476 (E.G.), DC04285 (B.W.S.), and EY17606 (A.P.S.), the Alfred P. Sloan Foundation (J.H.S.), the German Research Foundation (T.M.), the German Ministry for Education and Research (T.M.), the Max Planck Society (T.M.), the European Commission (T.M.), a Charles A. King Fellowship (V.B.), and the Karl Kirschgessner Foundation (A.P.S.).

Correspondence should be addressed to Alapakkam P. Sampath, Zilkha Neurogenetic Institute, USC Keck School of Medicine, 1501 San Pablo Street, ZNI 435, Los Angeles, CA 90089. E-mail: asampath@usc.edu.

D0I:10.1523/JNEUROSCI.3346-09.2009

Copyright $\odot 2009$ Society for Neuroscience ～0270-6474/09/2912802-05\$15.00/0 range of physical stimuli. Specifically, our discussion is focused on thresholding functions that reduce synaptic noise at retinal synapses subserving rod vision, active zone (AZ) specializations that enhance the temporal fidelity of transmission at cochlear hair cell synapses, and synaptic interactions within microcircuits postsynaptic to olfactory receptor cells.

Nonlinear properties of visual synapses improve the fidelity of the single photon response

A single ganglion cell - the output cell of the vertebrate retinareliably generates action potentials in response to the absorption of a single photon by a single rod photoreceptor within its receptive field (Barlow et al., 1971). Indeed, the absorption of just a few photons falling on the entire retina generates a visual percept (Hecht et al., 1942). The sensitivity of single ganglion cells is ensured by the profound convergence of rod outputs onto ganglion cells: a single ganglion cell may receive input from upward of 10,000 rods. Although this convergence ensures efficient photon detection, it presents the retinal circuitry with a significant problem: all rods generate transduction noise (arising from thermal isomerization of rhodopsin and the spontaneous activation of cGMP phosphodiesterase in the outer segment) (Field et al., $2005)$ that can be conveyed across the synapse. How can a ganglion cell separate a signal arising from one rod from noise generated by thousands of others? It has been appreciated for years that the retinal circuitry cannot simply combine the output of rods linearly: this would sum the noise and overwhelm the signal (Baylor et al., 1984; van Rossum and Smith, 1998). Rather, rod noise must be removed or attenuated by the interneurons that 
convey the rod output to ganglion cells by a nonlinear combination of rod outputs.

In the retina, mechanisms at the first two synapses in the rod pathway allow preferential transmission of the single photon response in lieu of noise generated by phototransduction and synaptic transmission, thereby improving the fidelity of signaling. First, at the rod-to-rod bipolar synapse, a threshold arising from saturation within the postsynaptic signaling cascade allows the circuitry to eliminate noise from the vast majority of rods not absorbing light. Second, at the rod bipolar-to-AII amacrine cell synapse, coordinated multivesicular release (MVR) likely allows the magnitude of single photon-evoked responses to exceed synaptic noise.

At the very first synapse of the mammalian visual system 20100 rods converge onto specialized rod "On" bipolar cells. Near absolute visual threshold perhaps one rod in this pool may have absorbed a photon. To make this situation worse, the small graded potential generated by photon absorption in rods $(\sim 1$ $\mathrm{mV}$ ) (Schneeweis and Schnapf, 1995) must overcome fluctuations produced by noise caused by fluctuations in the outer segment cGMP concentration. This noise is significant in that its amplitude overlaps considerably with the distribution of single photon response amplitudes. Under these circumstances, the detection of light can be improved by a nonlinear threshold between rods and rod bipolar cells that retains signals in rods absorbing light and eliminates noise in the remainder (van Rossum and Smith, 1998). Field and Rieke (2002) demonstrated that such processing occurs in the mouse retina, with the nonlinear threshold optimally positioned between the distribution of dark noise and single photon response amplitudes to separate single photon responses from the rod noise. In effect, this threshold eliminates rod bipolar responses to small fluctuations in rod voltage. Although this threshold has the consequence of eliminating the majority of postsynaptic responses to small, true signals, it provides more than a 300-fold improvement in the signal-to-noise ratio of the single photon response compared with the linear combination of rod outputs. Thus, this nonlinear threshold is critical for maximizing the signal-to-noise ratio of the single photon response near absolute threshold.

Since the processes that lead to neurotransmitter release from rods operate in their linear range, such a nonlinear threshold must occur postsynaptically in the rod bipolar dendrites (van Rossum and Smith, 1998; Thoreson et al., 2004). Rods are relatively depolarized in darkness, resulting in the continuous exocytosis of glutamatergic vesicles and the maintenance of a relatively high (tens of $\mu \mathrm{M}$; Hasegawa et al., 2006) steady-state cleft [glutamate]. Glutamate binds to metabotropic receptors (mGluR6) on rod bipolar dendrites and via activation of a $G_{o} \alpha$-coupled signaling pathway inhibits a membrane cation conductance. Recent evidence suggests that this conductance may in part be mediated by TRPM1 channels (Bellone et al., 2008; Shen et al., 2009). The hyperpolarization of rods by light leads to a reduction in glutamate release, which reduces the activity of the mGluR6 cascade and opens transduction channels thereby depolarizing the rod bipolar cell. Previous work has shown that nonlinear threshold is produced by saturation within the mGluR6 signaling cascade, not saturation of the mGluR6 receptors themselves (Sampath and Rieke, 2004). The level of activity of the mGluR6 signaling cascade is sufficiently high that nearly all the transduction channels are closed (Sampath and Rieke, 2004); thus, small fluctuations in synaptic glutamate and mGluR6 activity will not appreciably change the number of open transduction channels. Only larger reductions in glutamate that more likely result from photon ab- sorption will relieve saturation within the mGluR6 cascade and open transduction channels. Thus, saturation will act like a nonlinear threshold, and the level of saturation will determine the position of this threshold with respect to the distributions of noise and single photon response amplitudes. It remains to be seen how the extent of synaptic saturation is tuned to separate optimally the single photon responses from noise in the receptor cells.

Rod bipolar cells transfer the thresholded rod output to an interneuron called the AII amacrine cell. A single AII receives input from tens of rod bipolars, and when photons are scarce, the AII faces the same signal processing problem as the rod bipolar cell: how to separate a signal arising in one presynaptic neuron from the noise generated by synapses arising from the others? It has been suggested that the rod bipolar-AII synapse should perform a thresholding function as the rod-rod bipolar synapse does (Field and Rieke, 2002). MVR, or the concomitant exocytosis of the contents of multiple vesicles from the same presynaptic active zone, may serve as a presynaptic thresholding mechanism in the rod bipolar cell terminal.

Rod bipolar cell active zones are capable of highly synchronized MVR (2-4 vesicles/AZ/ms) (Singer et al., 2004). Importantly, the postsynaptic AMPA receptors in the AII are well suited to encode MVR: they have a low affinity for glutamate, exhibit rapid deactivation kinetics, and desensitize slowly relative to deactivation (Mørkve et al., 2002; Veruki et al., 2003). Thus, relatively high rates of sustained exocytosis ( 0.05 vesicle/AZ/ms) do not lead to postsynaptic receptor desensitization (Singer et al., 2004; Singer and Diamond, 2006). Additional evidence indicates that these postsynaptic receptors can encode MVR linearly: the amplitudes of postsynaptic currents varies directly with the number of released vesicles (Singer and Diamond, 2006), and the distribution of multi-quantal postsynaptic currents is described by binomial statistics (Singer et al., 2004).

MVR likely endows the rod bipolar synapse with a presynaptic thresholding function by ensuring that the majority of evoked synaptic events (arising from light-evoked depolarization of the rod bipolar cell) are larger than spontaneous synaptic noise, which is largely univesicular (Singer et al., 2004). Given that the coordinated release of multiple vesicles from the same presynaptic active zone occurs also at auditory and vestibular hair cell ribbon synapses (see below) (Glowatzki and Fuchs, 2002; Li et al., 2009), it is of interest to consider the mechanism(s) that may underlie it.

It is tempting to postulate that coordinated MVR reflects the release of multiple vesicles triggered by $\mathrm{Ca}^{2+}$ influx through a single open Ca channels in close proximity to both (giving rise to a small, precisely localized $\left[\mathrm{Ca}^{2+}\right]$ microdomain). At rod bipolar active zones, presynaptic Ca channels are quite close to release sites (Singer and Diamond, 2003), and the opening of one or few Ca channels can elicit exocytosis from rod bipolar and cochlear hair cell active zones (T. Jarsky and J. Singer, unpublished observations) (Brandt et al., 2005) (but see Coggins and Zenisek, 2009). Coordinated MVR, however, is observed during asynchronous release following the closure of $\mathrm{Ca}$ channels at rod bipolar cell synapses (Singer et al., 2004). As $\left[\mathrm{Ca}^{2+}\right]$ gradients dissipate rapidly following Ca channel closure, it is unlikely that a $\left[\mathrm{Ca}^{2+}\right]$ microdomain shared between two release sites is the only mechanism that triggers MVR. $\mathrm{Ca}^{2+}$-induced $\mathrm{Ca}^{2+}$ release from internal stores has been shown to evoke MVR at some synapses (Llano et al., 2000; Suryanarayanan and Slaughter, 2006), but evidence for such a mechanism is lacking for ribbon synapse of rod bipolar cells (Jarsky and Singer, unpublished observations). 
Thus, the mechanistic underpinning of MVR at ribbon synapses generally and the rod bipolar cell synapses specifically remain to be determined. It is likely, however, that MVR serves as a mechanism by which presynaptic activity is thresholded to permit the selective transmission of signals rather than synaptic noise.

\section{Fine-tuning exocytosis to make temporal encoding of sound precise}

The dynamics of sound encoding at the hair cell synapse play a key role in determining auditory representations in the CNS. Recordings along the auditory pathway indicate that the release of neurotransmitter from hair cells must exhibit fast kinetics to follow acoustic stimuli accurately with a periodicity of $<1 \mathrm{~ms}$. This temporal fidelity is maintained as acoustic signals vary greatly in intensity. Here, we will discuss recent work aimed at understanding quantitatively how the temporal precision of signaling is maintained at the first synapse in the auditory system in the face of noise generated by the stochastic nature of channel opening and exocytosis.

The inner hair cells (IHCs) of the cochlea transduce sound into a graded membrane potential changes and transmit this signal to the brain via their synapses with auditory nerve afferents. It is believed that an individual IHC drives auditory nerve fibers that differ in spontaneous and evoked rates, sound threshold, and dynamic range (Liberman, 1980). These different fibers collectively encode the large range of perceived sound pressures. Both presynaptic and postsynaptic mechanisms may underlie this heterogeneity in auditory nerve fiber spiking (Merchan-Perez and Liberman, 1996; Ruel et al., 2001). Transmission at IHC synapses relies on $\mathrm{Ca}_{\mathrm{V}} 1.3$ (L-type) Ca channels (Platzer et al., 2000; Brandt et al., 2003; Dou et al., 2004). These $\mathrm{Ca}_{\mathrm{V}} 1.3$ channels cluster at the presynaptic active zone, in discs of variable size $(420 \pm 130 \mathrm{~nm})$ and immunofluorescence intensity $(\mathrm{CV}=0.25)$ (Frank et al., 2009). This anatomical variability is reflected in functional variability in the magnitude of $\mathrm{Ca}^{2+}$ influx. Visualization of $\mathrm{Ca}^{2+}$ concentration between ribbon-type active zones reveals pronounced heterogeneity in the amplitude and voltage dependence of individual presynaptic $\left[\mathrm{Ca}^{2+}\right]$ microdomains- even within a given hair cell (Frank et al., 2009). $\mathrm{Ca}^{2+}$ microdomain amplitudes were correlated with the estimated synaptic ribbon size, which is a reflection of active zone size (Martinez-Dunst et al., 1997). Thus, individual hair cell active zones may differ in the number and voltage dependence of expressed Ca channels, which may mechanistically underlie divergent response properties of the postsynaptic auditory neurons (Frank et al., 2009). Indeed, a simple model of sound encoding qualitatively reproduces spiking with high spontaneous rate, low threshold, and small dynamic range for synapses with $50 \mathrm{Ca}$ channels and more, and predicts that low numbers of channels result in low spontaneous rates and high thresholds but wider dynamic ranges (T. Frank, N. Chapochnikov, A. and Neef, T. Moser, unpublished observations). How such presynaptic heterogeneity is established in the geometrically compact IHC remains to be studied.

Previous work estimated that the mean number of $\mathrm{Ca}_{\mathrm{V}} 1.3$ channels per synapse is $\sim 80$, each with a maximum open probability of 0.4 (Brandt et al., 2005; Meyer et al., 2009). Based on several lines of experimental evidence, exocytosis of each vesicle within the readily releasable pool (fully occupied: $\sim 30$ vesicles) may be controlled by $\mathrm{Ca}^{2+}$ influx through only one or few $\mathrm{Ca}$ channels located within tens of nanometers of a releasable vesicle $\left(\mathrm{Ca}^{2+}\right.$ nanodomain control; Brandt et al., 2005; Moser et al., 2006; Goutman and Glowatzki, 2007). With such tight functional coupling of $\mathrm{Ca}_{\mathrm{V}} 1.3$ channels and vesicle release sites, the synapse probably provides a saturating $\left[\mathrm{Ca}^{2+}\right]$ to the synaptic vesicle, thereby promoting rapid exocytosis (Beutner et al., 2001). The fast onset of exocytosis minimizes the response delay and jitter and consequentially generates functional "release units" that are effectively controlled by $\mathrm{Ca}^{2+}$ channel gating. Parallel release from multiple such units ensures high rates of release and high temporal precision of sound encoding (Khimich et al., 2005; Moser et al., 2006; Wittig and Parsons, 2008). Although this model readily explains several crucial aspects of sound encoding, more experiments are required to test its validity.

Synaptic transmission at hair cell ribbon synapses is mediated by AMPA receptors and similar to rod bipolar cell synapses, operates by MVR (Glowatzki and Fuchs, 2002; Singer et al., 2004; Keen and Hudspeth, 2006; Goutman and Glowatzki, 2007; Li et al., 2009). As a result of this MVR, EPSCs vary widely in amplitude-from tens to hundreds of picoampere-reflecting variability in the number of vesicles released (on average 2-4 and on rare occasions up to 20, depending on species, age, and the individual synapse) (Glowatzki and Fuchs, 2002; Keen and Hudspeth, 2006; Neef et al., 2007; Li et al., 2009). This variability in EPSC amplitude reflects significant differences in cleft [glutamate] (Li et al., 2009).

What is the advantage of synchronous release of multiple vesicles at the hair cell synapse? Multivesicular release likely increases the fidelity and temporal precision of sound encoding, as illustrated by a consideration of the IHC afferent synapse. Each IHC is innervated by 10-20 auditory nerve fibers, each of which receives only a single input from a single active zone in a single hair cell. Thus, the action potentials in the auditory nerve that drive sound perception each arise from the activity of only a single synapse. To make this synapse reliable (i.e., to allow deflections in the bundle of presynaptic stereocilia to evoke action potentials), EPSPs must be large. Thus, MVR may ensure that EPSPs reach the threshold for action potential generation. Also, larger EPSPs rise more quickly than slower ones and reach action potential threshold earlier, thereby reducing jitter in action potential timing.

But MVR is not always tightly coordinated: although there is little variability in EPSC waveforms at frog auditory hair cell synapses (Keen and Hudspeth, 2006; Li et al., 2009), EPSC waveforms recorded in murine auditory nerve afferents appear with different shapes. EPSCs either exhibit sharp rise times and monoexponential decays (monophasic) or appear quite variable in waveform with inflections during rise and decay phases (multiphasic) (Glowatzki and Fuchs, 2002; Li et al., 2009). Multiphasic events have slower rise times, most likely due to less synchronized release of multiple vesicles (L. Grant, E. Yi, and E. Glowatzki, unpublished observations) and recordings often show a considerable percentage of multiphasic events, sometimes larger than $50 \%$. Although it is unclear whether multiphasic EPSCs are simply a byproduct of MVR or whether they serve a role in coding specific aspects of sound, these events are unlikely to enhance precision in timing. Furthermore, EPSP amplitude distributions vary over a wide range and include small, subthreshold events (E. Yi, I. Roux, and E. Glowatzki, unpublished observations). It is interesting to consider the possibility that modulation of the postsynaptic conductances permits regulation of postsynaptic action potential generation by altering the EPSP waveform. Synaptic input from efferent fibers directly onto the IHC afferent dendrite (Liberman et al., 1990) could serve such a modulatory role. 


\section{Inhibition in local circuits improves the fidelity of olfactory signals in vertebrates and invertebrates}

In the olfactory systems of both vertebrates and invertebrates, inhibitory local circuits play a fundamental role in shaping the responses of second-order cells to excitatory input generated by action potentials from olfactory receptor neurons (ORNs) (Hamilton and Kauer, 1989; Olsen and Wilson, 2008). In the vertebrate olfactory bulb, the second-order mitral cells receive inhibitory input at two distinct regions of their dendritic tree: from periglomerular cells that innervate the distal tuft of the apical dendrite, and from axonless granule cells that form reciprocal dendrodendritic synapses with lateral dendrites in the external plexiform layer (Firestein, 2001). The specific functions performed by these two sets of interneurons are only partially understood. Computational studies, however, suggest that glomerular layer interneurons that contact the apical tufts of mitral cells function to provide gain control (Olsen and Wilson, 2008), expanding the dynamic range of olfactory system, and granule cell input helps decorrelate overlapping input patterns evoked by similar odorants (Yokoi et al., 1995).

While the organization of the inhibitory output connections from glomerular and granule cell layer interneurons are beginning to be understood, much less is known about the synaptic organization of excitatory inputs to these interneurons. Granule cells receive at least two types of excitatory input, based on morphological differences between spines on proximal and distal dendritic segments (Price and Powell, 1970). Recently, twophoton guided microstimulation was used to activate selectively proximal or distal excitatory inputs to granule cells recorded in rat olfactory bulb slices (Balu et al., 2007). $\mathrm{Ca}^{2+}$-imaging studies demonstrated that this method enables small groups of excitatory inputs to be activated when a dye-filled glass stimulating electrode is placed near (typically $10-20 \mu \mathrm{m}$ away) visualized dendritic spines. Weak stimulation (evoking both EPSPs and failures) near distal dendrites evoked relatively slow-rising EPSPs that depressed with paired stimulation. In contrast, weak stimulation near proximal granule cell dendrites evoked fast-rising EPSPs that facilitated with paired stimulation. While slower EPSP kinetics would be expected for distal inputs, reflecting the greater electrotonic filtering of those inputs with distance, the different forms of short-term plasticity indicated that granule cell receive two different functional types of excitatory input.

The origin of the proximal, facilitating input to granule cells also has been studied using a combined olfactory bulb/anterior piriform cortex (APC) slice preparation that partially maintained these pathways (Balu et al., 2007). Antidromic activation of olfactory bulb mitral cells by stimulation of the lateral olfactory tract (LOT) in the APC evoked slow-rising EPSPs that depressed in granule cells. Stimulation in the deep layers of APCs evoked fast-rising EPSPs in granule cells that facilitated with paired stimulation. These results suggest that feedback (or "centrifugal") projections from pyramidal cells in APC innervate proximal dendrites of granule cells. More recently, two-photon guided focal stimulation was used to demonstrate spike timing-dependent plasticity on the feedback projection from APC to granule cells, suggesting that plasticity in the synaptic connections between the olfactory bulb and APC may underlie some forms of olfactory learning (Gao and Strowbridge, 2009).

Similar local circuit interactions can be studied in the Drosophila olfactory system, in which the antennal lobe has become a powerful model for investigating the efficiency of coding. In the antennal lobe, analogous to the vertebrate olfactory bulb, recordings can be targeted to identified neurons labeled with fluorescent genetically encoded markers, thereby yielding insight into its anatomical organization (Bargmann, 2006). Just as for the vertebrates, each ORN expresses a single odorant receptor gene, and the axons of ORNs expressing the same receptor project to the same glomerulus in the antennal lobe. Here, ORN axons make excitatory synapses with second-order projection neurons (PNs) that send olfactory information to higher brain regions. Each PN arborizes in a single glomerulus, and thus receives direct ORN input from a single ORN type.

The systematic comparison of responses from the first-order ORNs and second order PNs allows the elucidation of basic principles of information transmission in early olfactory circuits. Activation of individual ORN types with specific odorants (called "private stimuli") has allowed the measurement of the purely intra-glomerular transformation between ORNs and their postsynaptic PNs (Bhandawat et al., 2007). This study found that the intra-glomerular transformation is well described by a boosting function that selectively amplifies weak odor responses. By blending private stimuli with varying concentrations of "public" stimuli that activate many ORN types, the effect of increasing activity in other glomeruli on the response of a PN to its cognate ORNs could be assessed. The effect of increasing activity in other glomeruli is to suppress the odor response such that the PN response saturates at higher input levels. Strikingly, the magnitude of the suppression depends solely on the total ORN activity.

The cellular mechanism for this boosting transformation within a glomerulus and inter-glomerular inhibition are well understood. The boosting transformation likely results from the large convergence between the ORNs and PNs. As many as 40-60 ORNs converge on to a single PN (Kazama and Wilson, 2009). Because ORN-PN synapses are strong and strongly depressing (Kazama and Wilson, 2008), weak odor responses are amplified selectively. The mechanistic basis for inter-glomerular inhibition is presynaptic inhibition at the ORN axon terminals (Olsen and Wilson, 2008), which is consistent with the observation that this form of inhibition is well described as "input gain control." These two mechanisms allow the efficient coding in the PN layer by allowing that each neuron to use its dynamic range uniformly and by permitting responses in different neurons to be statistically independent. The intra-glomerular boosting transformation creates $\mathrm{PN}$ responses that use their available dynamic range more uniformly, while inter-glomerular inhibition decorrelates PNs leading to greater statistical independence.

\section{Conclusion}

The work described at this Mini-Symposium highlight particular synaptic mechanisms in the visual, auditory, and olfactory system that addresses signal-processing problems faced by sensory circuits. No mechanism is the exclusive domain of a single sensory system. Rather, all systems use a combination of strategies including fine-tuning the dynamics of exocytosis, the responsiveness of postsynaptic neurons, and localized postsynaptic circuit interactions to match the downstream circuitry with the properties of the sensory receptor output over a wide range of stimulus intensities. This continuous matching of presynaptic and postsynaptic responsiveness ensures that changes in the physical world are encoded efficiently.

\section{References}

Balu R, Pressler RT, Strowbridge BW (2007) Multiple modes of synaptic excitation of olfactory bulb granule cells. J Neurosci 27:5621-5632.

Bargmann CI (2006) Comparative chemosensation from receptors to ecology. Nature 444:295-301. 
Barlow HB, Levick WR, Yoon M (1971) Responses to single quanta of light in retinal ganglion cells of the cat. Vision Res [Suppl 3]:87-101.

Baylor DA, Nunn BJ, Schnapf JL (1984) The photocurrent, noise and spectral sensitivity of rods of the monkey Macaca fascicularis. J Physiol 357:575-607.

Bellone RR, Brooks SA, Sandmeyer L, Murphy BA, Forsyth G, Archer S, Bailey E, Grahn B (2008) Differential gene expression of TRPM1, the potential cause of congenital stationary night blindness and coat spotting patterns (LP) in the Appaloosa horse (Equus caballus). Genetics 179:1861-1870.

Beutner D, Voets T, Neher E, Moser T (2001) Calcium dependence of exocytosis and endocytosis at the cochlear inner hair cell afferent synapse. Neuron 29:681-690.

Bhandawat V, Olsen SR, Gouwens NW, Schlief ML, Wilson RI (2007) Sensory processing in the Drosophila antennal lobe increases reliability and separability of ensemble odor representations. Nat Neurosci 10:1474-1482.

Brandt A, Striessnig J, Moser T (2003) CaV1.3 channels are essential for development and presynaptic activity of cochlear inner hair cells. J Neurosci 23:10832-10840.

Brandt A, Khimich D, Moser T (2005) Few CaV1.3 channels regulate the exocytosis of a synaptic vesicle at the hair cell ribbon synapse. J Neurosci 25:11577-11585.

Coggins M, Zenisek D (2009) Evidence that exocytosis is driven by calcium entry through multiple calcium channels in goldfish retinal bipolar cells. J Neurophysiol 101:2601-2619.

Dou H, Vazquez AE, Namkung Y, Chu H, Cardell EL, Nie L, Parson S, Shin HS, Yamoah EN (2004) Null mutation of alpha1D Ca ${ }^{2+}$ channel gene results in deafness but no vestibular defect in mice. J Assoc Res Otolaryngol 5:215-226.

Field GD, Rieke F (2002) Nonlinear signal transfer from mouse rods to bipolar cells and implications for visual sensitivity. Neuron 34:773-785.

Field GD, Sampath AP, Rieke F (2005) Retinal processing near absolute threshold: from behavior to mechanism. Annu Rev Physiol 67:491-514.

Firestein S (2001) How the olfactory system makes sense of scents. Nature 413:211-218.

Frank T, Khimich D, Neef A, Moser T (2009) Mechanisms contributing to synaptic $\mathrm{Ca}^{2+}$ signals and their heterogeneity in hair cells. Proc Natl Acad Sci U S A 106:4483-4488.

Gao Y, Strowbridge BW (2009) Long-term plasticity of excitatory inputs to granule cells in the rat olfactory bulb. Nat Neurosci 12:731-733.

Glowatzki E, Fuchs PA (2002) Transmitter release at the hair cell ribbon synapse. Nat Neurosci 5:147-154.

Goutman JD, Glowatzki E (2007) Time course and calcium dependence of transmitter release at a single ribbon synapse. Proc Natl Acad Sci U S A 104:16341-16346.

Hamilton KA, Kauer JS (1989) Patterns of intracellular potentials in salamander mitral/tufted cells in response to odor stimulation. J Neurophysiol 62:609-625.

Hasegawa J, Obara T, Tanaka K, Tachibana M (2006) High-density presynaptic transporters are required for glutamate removal from the first visual synapse. Neuron 50:63-74.

Hecht S, Schlaer S, Pirenne MH (1942) Energy, quanta, and vision. J Gen Physiol 25:819-840.

Kazama H, Wilson RI (2008) Homeostatic matching and nonlinear amplification at identified central synapses. Neuron 58:401-413.

Kazama H, Wilson RI (2009) Origins of correlated activity in an olfactory circuit. Nat Neurosci 12:1136-1144.

Keen EC, Hudspeth AJ (2006) Transfer characteristics of the hair cell's afferent synapse. Proc Natl Acad Sci U S A 103:5537-5542.

Khimich D, Nouvian R, Pujol R, Tom Dieck S, Egner A, Gundelfinger ED, Moser T (2005) Hair cell synaptic ribbons are essential for synchronous auditory signalling. Nature 434:889-894.

Li GL, Keen E, Andor-Ardó D, Hudspeth AJ, von Gersdorff H (2009) The unitary event underlying multiquantal EPSCs at a hair cell's ribbon synapse. J Neurosci 29:7558-7568.

Liberman MC (1980) Efferent synapses in the inner hair cell area of the cat cochlea: an electron microscopic study of serial sections. Hear Res 3:189-204.

Liberman MC, Dodds LW, Pierce S (1990) Afferent and efferent innervation of the cat cochlea: quantitative analysis with light and electron microscopy. J Comp Neurol 301:443-460.

Llano I, González J, Caputo C, Lai FA, Blayney LM, Tan YP, Marty A (2000) Presynaptic calcium stores underlie large-amplitude miniature IPSCs and spontaneous calcium transients. Nat Neurosci 3:1256-1265.

Martinez-Dunst C, Michaels RL, Fuchs PA (1997) Release sites and calcium channels in hair cells of the chick's cochlea. J Neurosci 17:9133-9144.

Merchan-Perez A, Liberman MC (1996) Ultrastructural differences among afferent synapses on cochlear hair cells: correlations with spontaneous discharge rate. J Comp Neurol 371:208-221.

Meyer AC, Frank T, Khimich D, Hoch G, Riedel D, Chapochnikov NM, Yarin YM, Harke B, Hell SW, Egner A, Moser T (2009) Tuning of synapse number, structure and function in the cochlea. Nat Neurosci 12:444-453.

Mørkve SH, Veruki ML, Hartveit E (2002) Functional characteristics of non-NMDA-type ionotropic glutamate receptor channels in AII amacrine cells in rat retina. J Physiol 542:147-165.

Moser T, Neef A, Khimich D (2006) Mechanisms underlying the temporal precision of sound coding at the inner hair cell ribbon synapse. J Physiol 576:55-62.

Neef A, Khimich D, Pirih P, Riedel D, Wolf F, Moser T (2007) Probing the mechanism of exocytosis at the hair cell ribbon synapse. J Neurosci 27:12933-12944.

Olsen SR, Wilson RI (2008) Lateral presynaptic inhibition mediates gain control in an olfactory circuit. Nature 452:956-960.

Platzer J, Engel J, Schrott-Fischer A, Stephan K, Bova S, Chen H, Zheng H, Striessnig J (2000) Congenital deafness and sinoatrial node dysfunction in mice lacking class D L-type $\mathrm{Ca}^{2+}$ channels. Cell 102:89-97.

Price JL, Powell TP (1970) The morphology of the granule cells of the olfactory bulb. J Cell Sci 7:91-123.

Ruel J, Nouvian R, Gervais d'Aldin C, Pujol R, Eybalin M, Puel JL (2001) Dopamine inhibition of auditory nerve activity in the adult mammalian cochlea. Eur J Neurosci 14:977-986.

Sampath AP, Rieke F (2004) Selective transmission of single photon responses by saturation at the rod-to-rod bipolar synapse. Neuron 41:431-443.

Schneeweis DM, Schnapf JL (1995) Photovoltage of rods and cones in the macaque retina. Science 268:1053-1056.

Shen Y, Heimel JA, Kamermans M, Peachey NS, Gregg RG, Nawy S (2009) A transient receptor potential-like channel mediates synaptic transmission in rod bipolar cells. J Neurosci 29:6088-6093.

Singer JH, Diamond JS (2003) Sustained $\mathrm{Ca}^{2+}$ entry elicits transient postsynaptic currents at a retinal ribbon synapse. J Neurosci 23:10923-10933.

Singer JH, Diamond JS (2006) Vesicle depletion and synaptic depression at a mammalian ribbon synapse. J Neurophysiol 95:3191-3198.

Singer JH, Lassová L, Vardi N, Diamond JS (2004) Coordinated multivesicular release at a mammalian ribbon synapse. Nat Neurosci 7:826-833.

Suryanarayanan A, Slaughter MM (2006) Synaptic transmission mediated by internal calcium stores in rod photoreceptors. J Neurosci 26:1759-1766.

Thoreson WB, Rabl K, Townes-Anderson E, Heidelberger R (2004) A highly $\mathrm{Ca}^{2+}$-sensitive pool of vesicles contributes to linearity at the rod photoreceptor ribbon synapse. Neuron 42:595-605.

van Rossum MC, Smith RG (1998) Noise removal at the rod synapse of mammalian retina. Vis Neurosci 15:809-821.

Veruki ML, Mørkve SH, Hartveit E (2003) Functional properties of spontaneous EPSCs and non-NMDA receptors in rod amacrine (AII) cells in the rat retina. J Physiol 549:759-774.

Wittig JH Jr, Parsons TD (2008) Synaptic ribbon enables temporal precision of hair cell afferent synapse by increasing the number of readily releasable vesicles: a modeling study. J Neurophysiol 100:1724-1739.

Yokoi M, Mori K, Nakanishi S (1995) Refinement of odor molecule tuning by dendrodendritic synaptic inhibition in the olfactory bulb. Proc Natl Acad Sci U S A 92:3371-3375. 